\title{
Cultivo de tambaqui em canais de abastecimento sob diferentes densidades de peixes
}

\author{
Alinne Dué Ramos da SILVA¹, Robson Batista dos SANTOS², Arthur Murilo da Silva Souza BRUNO², \\ Emerson Carlos SOARES ${ }^{2}$
}

RESUMO

A piscicultura moderna baseia-se no melhor sistema de criação, qualidade ambiental e ganhos de produtividade. Neste experimento foi analisado o efeito da densidade de cultivo na qualidade da água, e posteriormente, no desempenho zootécnico dos tambaquis (Colossoma macropomum). Os juvenis de tambaqui com 13,6 \pm 4,1g de peso médio foram mantidos em canais de abastecimento com 40 metros de extensão, dividido em quinze seçóes de um metro cúbico. Foram testadas três densidades (60, 90 e 120 peixe $\left.\mathrm{m}^{-3}\right)$ em delineamento experimental inteiramente casualizado, com cinco repetiçóes. Os resultados dos parâmetros físico-químicos da água foram: temperatura variou de $28,0-31,7{ }^{\circ} \mathrm{C}$, o oxigênio dissolvido de $5,9-7,4 \mathrm{mg} \mathrm{L}^{-1}$, o $\mathrm{pH}$ de 6,8-7,5, a concentração média de amônia ficou ao redor de 0,1-0,3 $\mathrm{mg} \mathrm{L}^{-1}$. Os valores de alcalinidade e dureza foram de 24,0-34,0 $\mathrm{mg} \mathrm{L}^{-1}\left(\mathrm{CaCO}_{3}\right)$ e 24,0-37,0 $\mathrm{mg} \mathrm{L}^{-1}\left(\mathrm{CaCO}_{3}, \mathrm{MgCO}_{3}\right)$. A condutividade elétrica variou entre 0,07-106 $\mu \mathrm{S} \mathrm{cm}^{-1}$ e a concentração do total de sólidos dissolvidos ficou entre 18,37-53,00 $\mathrm{mg} \mathrm{L}^{-1}$. Já o ganho de peso final foi de 35,76, 32,95 e 36,25 g para as densidades de 60, 90 e 120 peixes $\mathrm{m}^{-3}$, respectivamente, não sendo verificadas diferenças significativas nos tratamentos avaliados. Os dados do presente estudo abrem precedentes para que cultivo de peixes em canais de irrigação possa ser integrado com outras culturas, maximizando o uso da água.

PALAVRAS-CHAVE: Piscicultura em canais, Qualidade da água, Colossoma macropomum.

\section{Tambaqui farming in irrigation channels under different fish densities}

\section{ABSTRACT}

Modern fish farming is based upon best-practice breeding technology, environmental quality and productivity benefit. In this experiment we analyzed fish density effects on water quality, and afterwards on breeding efficiency of Tambaqui (Colossoma macropomum). Juveniles with an average weight of 13,6 4,1 g were reared in $40 \mathrm{~m}$ long irrigation channels, which were divided in 15 sections of $1 \mathrm{~m}^{3}$ in each case. Three fish densities $\left(60,90\right.$ and 120 individuals per $\left.\mathrm{m}^{3}\right)$ were tested, with five replications. Water quality parameters were as follows: water temperature $28,0-31,7^{\circ} \mathrm{C}$, dissolved oxygen $5,9-7,4$ mg $\mathrm{L}^{-1}$, $\mathrm{pH}$ 6,8-7,5, ammonium concentration $0,1-0,3 \mathrm{mg} \mathrm{L}^{-1}$, alkalinity 24,0-34,0 $\mathrm{mg} \mathrm{L}^{-1}\left(\mathrm{CaCO}_{3}\right)$, hardness 24,0-37,0 mg L-1 $\left(\mathrm{CaCO}_{3}, \mathrm{MgCO}_{3}\right)$, conductivity $0,07-106 \mu \mathrm{S} \mathrm{cm}^{-1}$ and total dissolved solids $18,37-53,00 \mathrm{mg} \mathrm{L}^{-1}$. The increase in fish weight was $35,76,32,95$ and $36,25 \mathrm{~g}$ for fish density of 60,90 , and 120 individuals per $\mathrm{m}^{3}$, respectively. No difference among fish density was observed. In conclusion, there is the possibility of integrating the fish farming with other cultures, maximizing the use of water resources.

KEYWORDS: Fish cultures in channels, Water quality, Colossoma macropomum.

\footnotetext{
1 Programa de Pós-graduação em Diversidade Biológica e Conservação nos Trópicos, DiBiCT, ICBS/UFAL. Praça Afrânio Jorge, s/n. Centro, Prado, 57000-000, Maceió, AL, Brasil alinne_due@hotmail.com.

2 Departamento de Engenharia de Pesca/UFAL, campus Arapiraca-Pólo Penedo, Av. Beira Rio, S/n, 57200-000 - Penedo, AL, Brasil, rob.dja@bol.com.br arthurmurilo@gmail.com, soaemerson@gmail.com.
} 


\section{INTRODUÇÃO}

Com o desenvolvimento das fazendas de cultivos aquáticas tem-se observado uma tendência ao uso de sistemas de produçáo cada vez mais intensivos. Isto implica na adoção de um conjunto de práticas de manejo que melhorem a sustentabilidade destes criatórios, analisando os principais efeitos nas diversas fases de cultivo, principalmente com relação à frequência e privação alimentar, taxa de arraçoamento, renovação e qualidade da água, incremento da densidade de estocagem e modelo de área produtiva (Pereira et al. 2008). A solução para piscicultura moderna sustentável decorre em encontrar o equilíbrio entre o melhor sistema de criação, densidade ideal de produção, taxa de arraçoamento equilibrada e água com boa capacidade tamponante (Vallejo e González-Posada 2007).

No tocante aos sistemas de produção, a piscicultura em canais secundários atende os preceitos de atividades que promovem melhores ganhos de produtividade e uma melhor qualidade ambiental, pois apresenta construção de fácil execução e baixo custo; reduz o impacto ambiental; além de diminuir o estresse nas etapas de manejo, já que facilita o manuseio e monitoramento das condiçóes gerais dos organismos (Lima et al. 2008).

Estudos com espécies tropicais utilizando canais de irrigaçáo como ambiente de cultivo foram recomendados pela FAO (Organização das Naçôes Unidas para Alimentação e Agricultura). Por outro lado, o uso destas estruturas para produção de peixes oferece certos riscos e está sujeito às regras de gestáo hídrica, da qual são priorizadas as práticas agrícolas, onde problemas como uso de produtos químicos, elevado índice de evaporação, níveis de água irregulares e aumento da salinização e sedimentação são comuns neste tipo de sistema (Redding e Midlen 1992).

De certa forma, estes problemas parecem não ter afetado o desenvolvimento da atividade piscícola em canais de irrigação em Israel, já que, com a adequação da temporada de cultivo agrícola com a piscicultura em canais, conseguiu-se mitigar os possíveis conflitos decorrentes desta atividade na região (Redding e Midlen 1992).

Experimentos com peixes em canais apresentaram resultados satisfatórios (Arbeláez-Rojas et al. 2002; Lima et al. 2008), contudo, informaçôes referentes ao manejo alimentar e densidade de cultivo, fluxo e qualidade da água, bem como, tamanho e espaço ideal destinado à criaçáo neste tipo de ambiente divide opinióes entre técnicos, pesquisadores e produtores, possivelmente devido à carência de dados seguros, dificultando a sua aplicabilidade com fins aquícolas (Redding e Midlen 1992).

O tambaqui, Colossoma macropomum (Cuvier, 1816) é uma espécie largamente encontrada na América do Sul como peixe de cultivo, principalmente na região Norte e Nordeste. Por apresentar características como rusticidade, alta produtividade e aceitar raçôes extrusadas e peletizadas, este onívoro tornou-se uma espécie chave em programas de criação no Brasil (Araújo-Lima e Gomes 2010). Com um incremento de $87 \%$ na sua produção nos últimos três anos (MPA 2010), esta espécie se apresenta como uma forte candidata ao cultivo em viveiros ou locais com maior movimentaçâo de água pela facilidade de adaptação.

Um dos passos para verificar a eficiência de canais como ambiente para produção de tambaquis é a determinação da melhor densidade de cultivo, a qual é representada pela maior quantidade de indivíduos produzidos eficientemente por unidade de área, não afetando parâmetros biológicos e de qualidade de água, sendo estes fatores, possíveis causas no fracasso de sistemas confinados (Redding e Midlen 1992; Arbeláez-Rojas et al. 2002).

Objetivou-se, com o presente trabalho, estimar os efeitos da densidade de cultivo em canais de abastecimento na qualidade da água e no desempenho zootécnico de tambaquis.

\section{MATERIAL E MÉTODOS}

O estudo foi conduzido na Estaçáo de Piscicultura de Betume-CODEVASF (Companhia de Desenvolvimento dos Vales do São Francisco e do Parnaíba), localizada no município de Neópolis - SE, no período de 20 de março a 04 de maio de 2010 totalizando 45 dias.

Dois mil juvenis de tambaqui foram transportados em sacos plásticos de 30 litros, sendo utilizados $20 \%$ do seu volume preenchidos com cinco litros de água e o restante adicionados com oxigênio, contendo solução salina de $2 \mathrm{~g}$ de sal por litro de água para evitar a perda de eletrólitos dos indivíduos para o meio. Antes da biometria inicial 1080 exemplares, com 13,6 $\pm 4,1 \mathrm{~g}$ de peso médio inicial foram aclimatados e monitorados em quatro tanques-rede, com volume de quatro metros cúbicos, dispostos em um viveiro escavado, durante cinco dias, conforme sugerido por AraújoLima e Gomes (2010).

$\mathrm{O}$ experimento seguiu um delineamento experimental inteiramente casualizado, com três tratamentos e densidades de: 60 peixes $\mathrm{m}^{-3}, 90$ peixes $\mathrm{m}^{-3} \mathrm{e} 120$ peixes $\mathrm{m}^{-3}$, cada um com cinco repetiçōes, distribuídos aleatoriamente, totalizando 15 unidades experimentais ou secçóes de um metro cúbico cada, separadas por três zonas de alívio, correspondendo a duas vezes o tamanho da área de cada secçáo.

O canal de abastecimento, com $40 \mathrm{~m}$ de extensão, apresentava formato trapezoidal, de dimensōes $1,50 \mathrm{~m}$ altura x $1,40 \mathrm{~m}$ base maior $\mathrm{x} 0,35 \mathrm{~m}$ base menor, e vazáo controlada $\left(6.000 \mathrm{~L} \mathrm{~h}^{-1}\right)$. As telas de arame (quatro centímetros entre nós) usadas nas secçôes experimentais (volume útil de $0,90 \mathrm{~m}^{3}$ ) 
eram revestidas por zinco e PVC e costuradas em armação de aço, dispostas e niveladas no canal. Adicionalmente foram colocadas redes secundárias (tela de segurança e rede antipássaro) para retenção de peixes no caso de fuga ou predação. Durante o período experimental, as telas foram limpas semanalmente para evitar a formação do perifíton, retenção de detritos orgânicos e partículas inorgânicas associadas.

Os peixes foram medidos, pesados e distribuídos homogeneamente nas parcelas $(8,0 \pm 1,3 \mathrm{~cm}$ de comprimento médio padrão e 13,6 $\pm 4,1 \mathrm{~g}$ de peso médio), totalizando 1.080 exemplares. Os peixes foram alimentados com raçáo extrusada comercial Nutron, contendo 36\% (mínimo) de proteína bruta, umidade de 12\% (máximo), 4\% (mínimo) de extrato etéreo, 12\% (máximo) de matéria mineral, $8 \%$ (máximo) de material fibroso, $2 \%$ (máximo) de cálcio e $0,6 \%$ (mínimo) de fósforo. Para o fornecimento da ração foi levada em consideração à biomassa estocada, sendo fornecida uma porção diária total, em média, de $5 \%$ do peso vivo, administrados três vezes ao dia $(7: 30,11: 30$ e 16:30 h).

Os parâmetros de desempenho avaliados foram: ganho de peso (g) (GP): GP = peso médio final menos peso médio inicial; consumo médio individual (CMDi): $\mathrm{CMDi}$ = quantidade de ração fornecida por dia (g) dividida pelo número de peixes; consumo individual médio de ração no final do experimento (CIMFi): $\mathrm{CIMFi}$ = somatória do CMDi; Índice de conversão alimentar aparente (CAA): CAA = CIMFi dividido pelo peso médio final (g) menos o peso médio inicial (g); taxa de crescimento específico (\%) (CEP): CEP = 100 vezes o logaritmo neperiano do peso médio final $(\mathrm{g})$ menos o logaritmo neperiano do peso médio inicial (g) dividido pelo tempo (dias); taxa de sobrevivência dos peixes (\%) (TS): TS = 100 vezes o número final de peixes dividido pelo número inicial de peixes.
Com o propósito de analisar o efeito da densidade de estocagem sobre a qualidade da água, foram mensurados e monitorados diariamente, na profundidade de $0,5 \mathrm{~m}$ nas secçóes de cada bloco, em dois horários (8:00 h e 16:00 h), os seguintes parâmetros: temperatura $\left({ }^{\circ} \mathrm{C}\right)$ e oxigênio dissolvido (OD, $\mathrm{mg} \mathrm{L}^{-1}$ ), por meio de um oxímetro (YSI555, Incorporated Yellow Springs, USA.). Semanalmente foram avaliados: alcalinidade e dureza total $\left(\mathrm{CaCO}_{3}\right.$ e $\left.\mathrm{MgCO}_{3}\right)$ por titulometria, amônia total, de acordo com Standard MethodsAPHA (1995), pH, condutividade elétrica e sólidos dissolvidos totais foram avaliados semanalmente, por meio de sonda multi-parâmetros (Hanna, HI 9828, Woonsocket, USA.).

Após pesagem no início do experimento, a homogeneidade do lote de peixes foi comprovada pelo teste Cochran, $(P<0,05)$. Os resultados obtidos no estudo foram analisados pelo programa SYSTAT 10, por intermédio da análise de variância (ANOVA, $P<0,05$ ) com a finalidade de verificar o efeito dos tratamentos sobre o desempenho dos peixes e a qualidade da água. Para análise das variáveis limnológicas e de desempenho dos peixes, quando " $\mathrm{F}$ " foi significativo, utilizou-se teste Tukey $(P<0,05)$.

\section{RESULTADOS}

Não ocorreram diferenças significativas nos parâmetros de qualidade de água $(P>0,05)$, com relação à localização das unidades experimentais ao longo do canal de abastecimento, indicando que os efluentes provenientes das secçôes antes da área de alívio não influenciaram nas secções seguintes (Tabela 1).

Os valores médios das variáveis limnológicas observados em relação às semanas de cultivo e horários encontram-se dispostos na Tabela 2. A temperatura alcançou níveis médios durante os 45 dias do período experimental de 30,0 \pm 0,7 ${ }^{\circ} \mathrm{C}$. No horário das 16:00 horas foram encontrados valores de temperaturas significativamente maiores $\left(31,7^{\circ} \mathrm{C}\right) \mathrm{em}$

Tabela 1 - Valores médios ( \pm desvio-padrão) das variáveis temperatura e qualidade de água no cultivo de tambaqui em canais de abastecimento, nas diferentes secções durante os 45 dias de experimento(1). OD = 0xigênio dissolvido.

\begin{tabular}{lcccccc}
\hline \multirow{2}{*}{ Variáveis analisadas } & \multicolumn{2}{c}{ secção 1 } & \multicolumn{2}{c}{ secção 2 } & \multicolumn{2}{c}{ secção 3} \\
\cline { 2 - 7 } & Manhã & Tarde & Manhã & Tarde & Manhã & Tarde \\
\hline Temperatura ( $\left.{ }^{\circ} \mathrm{C}\right)$ & $29,1 \pm 0,8$ & $30,8 \pm 0,9$ & $29,1 \pm 0,7$ & $30,9 \pm 0,8$ & $29,5 \pm 0,6$ & $30,8 \pm 0,9$ \\
OD (mg L-1) & $6,8 \pm 0,52$ & $7,10 \pm 0,51$ & $6,6 \pm 0,5$ & $6,9 \pm 0,5$ & $6,5 \pm 0,5$ & $7,0 \pm 0,5$ \\
pH & $7,0 \pm 0,3$ & $7,0 \pm 0,4$ & $7,0 \pm 0,3$ & $7,1 \pm 0,4$ & $7,0 \pm 0,3$ & $7,1 \pm 0,4$ \\
Amônia (mg L-1) & $0,1 \pm 0,1$ & $0,1 \pm 0,1$ & $0,1 \pm 0,1$ & $0,1 \pm 0,1$ & $0,1 \pm 0,1$ & $0,1 \pm 0,1$ \\
Alcalinidade (mg L-1) & $28,4 \pm 5,1$ & $27,4 \pm 2,5$ & $29,3 \pm 4,4$ & $28,0 \pm 3,0$ & $28,0 \pm 4,0$ & $27,3 \pm 2,8$ \\
Dureza (mg L-1) & $31,6 \pm 10,0$ & $32,8 \pm 9,5$ & $28,2 \pm 8,6$ & $30,9 \pm 7,8$ & $28,0 \pm 5,2$ & $28,5 \pm 3,3$ \\
\hline
\end{tabular}

(1) Não foram detectadas diferenças significativas nas variáveis de qualidade de água com relação à localização das secções ao longo do canal de abastecimento, pelo teste de Tukey, a $5 \%$ de probabilidade. 
relação aos dados coletados às oito horas da manhã $\left(28,0^{\circ} \mathrm{C}\right)$. Os menores valores registrados nos dois horários ocorreram durante o vigésimo oitavo dia de cultivo $\left(28,0\right.$ e $29,8{ }^{\circ} \mathrm{C}$, respectivamente).

As concentraçóes de oxigênio dissolvido apresentaram um padrão similar ao da temperatura, sendo menor pela manhã $\left(5,9 \pm 0,3 \mathrm{mg} \mathrm{L}^{-1}\right)$ e maior no período da tarde $(7,4$ $\left.\pm 0,3 \mathrm{mg} \mathrm{L}^{-1}\right)$, com incremento gradual e lento durante o dia, até declinar em função da diminuição da incidência de luz disponível (Tabela 2). Valores de oxigênio dissolvido $\left(6,4 \pm 0,5 \mathrm{mg} \mathrm{L}^{-1}\right)$ só apresentaram diferenças significativas a partir da quarta semana do experimento, ocorrendo pequena diminuição nas suas concentraçóes à medida que o cultivo se prolongava.

Os dados de $\mathrm{pH}$ comprovaram não ocorrer alteraçóes severas no período de estudo, todavia, valores menores deste parâmetro foram encontrados no período da manhã e em maiores densidades. Náo ocorreram diferenças significativas nos valores de amônia entre os horários coletados e entre as densidades, porém foi observado um aumento pontual na quarta e quinta semana de estudo (Tabela 2).

Os valores de alcalinidade apresentaram diferenças estatísticas $(P<0,05)$ em relação ao tempo de cultivo. Maiores valores desta variável foram observados nas duas primeiras semanas de estudo, declinando ao longo do cultivo e atingindo teores mais baixos na quarta semana do experimento. Nas maiores densidades do experimento foram encontrados maiores valores de alcalinidade e dureza, entretanto, estas alteraçóes não foram significativas (Tabela 2).

Ao longo do período experimental a dureza da água não sofreu grandes alteraçóes, ocorrendo uma leve tendência de aumento na quarta e quinta semanas experimental $(P<0,05)$, retornando ao mesmo padrão das semanas anteriores, no período final do cultivo (Tabela 2).

Os valores médios dos parâmetros limnológicos observados em relação aos tratamentos, encontram-se dispostos na Tabela 3. Apesar de ocorrer um aumento significativo com relaçáo à primeira semana de cultivo $(P<0,05)$, os resultados de condutividade elétrica e sólidos dissolvidos totais, em geral, foram considerados dentro dos limites aceitáveis para criação da espécie (Tabela 3).

Os valores para o desenvolvimento zootécnico dos juvenis de tambaqui estão dispostos na Tabela 4. O ganho médio de peso dos peixes no presente trabalho foi de $0,93 \mathrm{~g} \mathrm{dia}^{-1}$. Apesar de não haver diferença significativa entre as densidades, observou-se que a quantidade de 90 peixes $\mathrm{m}^{-3}$ apresentou aproximadamente $80 \%$ de sobrevivência.

\section{DISCUSSÃO}

As áreas de alívio, responsáveis pela recuperação da água, e a vazão utilizada, podem ter mitigado os efeitos adversos provenientes de uma má qualidade limnológica no sistema de cultivo do presente estudo, condição esta observada nos experimentos de Lima et al. (2008).

Tabela 2 - Valores médios ( \pm desvio padrão) das variáveis temperatura e qualidade da água durante o cultivo de tambaqui em canais de abastecimento, em diferentes horários e semanas do período experimental ${ }^{(1)}$. $\mathrm{S}=$ Semana, $\mathrm{H}=$ Horário do dia, $\mathrm{T}=$ Temperatura $\left({ }^{\circ} \mathrm{C}\right), \mathrm{OD}=0$ xigênio dissolvido $\left(\mathrm{mg} \mathrm{L}^{-1}\right)$, $\mathrm{pH}$ $=$ Potencial hidrogênico, $\mathrm{NH}_{3}=$ Amônia $\left(\mathrm{mg} \mathrm{L}^{-1}\right)$, Alcal. = Alcalinidade $\left(\mathrm{mg} \mathrm{L}^{-1}\right)$, DT = Dureza total $\left(\mathrm{mg} \mathrm{L}^{-1}\right)$.

\begin{tabular}{llllllll}
\hline $\mathrm{S}$ & $\mathrm{H}$ & $\mathrm{T}\left({ }^{\circ} \mathrm{C}\right)$ & $\mathrm{OD}$ & $\mathrm{pH}$ & $\mathrm{NH}_{3}$ & Alcal. & $\mathrm{DT}$ \\
\hline \multirow{2}{*}{$1^{\mathrm{a}}$} & Manhã & $29,4 \pm 0,5^{\mathrm{aA}}$ & $7,1 \pm 0,2^{\mathrm{aA}}$ & $7,0 \pm 0,0^{\mathrm{aA}}$ & $0,1 \pm 0,0^{\mathrm{aA}}$ & $34,0 \pm 2,2^{\mathrm{aA}}$ & $24,0 \pm 0,0^{\mathrm{aA}}$ \\
& Tarde & $30,6 \pm 0,4^{\mathrm{bA}}$ & $7,4 \pm 0,2^{2 \mathrm{~A}}$ & $7,2 \pm 0,3^{\mathrm{aA}}$ & $0,1 \pm 0,0^{\mathrm{aA}}$ & $31,0 \pm 1,1^{\mathrm{bA}}$ & $28,0 \pm 0,0^{\mathrm{aA}}$ \\
\hline \multirow{2}{*}{$2^{\mathrm{a}}$} & Manhã & $29,4 \pm 0,4^{\mathrm{aA}}$ & $6,9 \pm 0,4^{\mathrm{aA}}$ & $7,2 \pm 0,3^{\mathrm{aB}}$ & $0,1 \pm 0,0^{\mathrm{aA}}$ & $30,0 \pm 2,4^{\mathrm{aA}}$ & $29,3 \pm 6,5^{\mathrm{aA}}$ \\
& Tarde & $31,7 \pm 0,6^{\mathrm{bB}}$ & $7,4 \pm 0,3^{\mathrm{bA}}$ & $7,2 \pm 0,3^{\mathrm{aB}}$ & $0,1 \pm 0,0^{\mathrm{aA}}$ & $28,0 \pm 0,0^{\mathrm{aB}}$ & $28,0 \pm 0,0^{\mathrm{aA}}$ \\
\hline \multirow{2}{*}{$3^{\mathrm{a}}$} & Manhã & $29,4 \pm 0,3^{\mathrm{aA}}$ & $6,8 \pm 0,3^{\mathrm{aA}}$ & $7,0 \pm 0,0^{\mathrm{aA}}$ & $0,1 \pm 0,0^{\mathrm{aA}}$ & $28,0 \pm 0,0^{\mathrm{aA}}$ & $28,0 \pm 0,0^{\mathrm{aA}}$ \\
& Tarde & $30,8 \pm 0,8^{\mathrm{bA}}$ & $7,2 \pm 0,2^{\mathrm{bA}}$ & $7,0 \pm 0,0^{\mathrm{aA}}$ & $0,1 \pm 0,0^{\mathrm{aA}}$ & $28,0 \pm 0,0^{\mathrm{aA}}$ & $28,0 \pm 0,0^{\mathrm{aA}}$ \\
\hline \multirow{2}{*}{$4^{\mathrm{a}}$} & Manhã & $28,0 \pm 0,8^{\mathrm{aB}}$ & $6,4 \pm 0,5^{\mathrm{aB}}$ & $6,8 \pm 0,3^{\mathrm{aA}}$ & $0,3 \pm 0,0^{\mathrm{aB}}$ & $24,7 \pm 1,6^{\mathrm{aBA}}$ & $26,0 \pm 2,3^{\mathrm{aA}}$ \\
& Tarde & $29,8 \pm 0,9^{\mathrm{bA}}$ & $6,7 \pm 0,5^{\mathrm{aB}}$ & $7,0 \pm 0,3^{\mathrm{aA}}$ & $0,3 \pm 0,0^{\mathrm{aB}}$ & $24,0 \pm 0,0^{\mathrm{aBA}}$ & $32,0 \pm 4,4^{\mathrm{bA}}$ \\
\hline \multirow{2}{*}{$5^{\mathrm{a}}$} & Manhã & $29,5 \pm 0,5^{\mathrm{aA}}$ & $6,5 \pm 0,3^{\mathrm{aB}}$ & $6,8 \pm 0,3^{\mathrm{aA}}$ & $0,2 \pm 0,1^{a^{\mathrm{AA}}}$ & $26,3 \pm 2,2^{\mathrm{aA}}$ & $37,0 \pm 14,2^{\mathrm{aA}}$ \\
& Tarde & $31,1 \pm 0,4^{\mathrm{bA}}$ & $6,7 \pm 0,3^{\mathrm{aB}}$ & $6,8 \pm 0,3^{\mathrm{aA}}$ & $0,2 \pm 0,1^{\text {aA }}$ & $27,0 \pm 1,0^{\mathrm{aB}}$ & $37,0 \pm 14,2^{\mathrm{aA}}$ \\
\hline \multirow{2}{*}{$6^{\mathrm{a}}$} & Manhã & $29,2 \pm 0,3^{\mathrm{aA}}$ & $5,9 \pm 0,3^{\mathrm{aB}}$ & $7,5 \pm 0,0^{\mathrm{aB}}$ & $0,1 \pm 0,0^{\mathrm{aA}}$ & $28,0 \pm 0,0^{\mathrm{aA}}$ & $28,0 \pm 0,0^{\mathrm{aA}}$ \\
& Tarde & $31,0 \pm 0,7^{\mathrm{bA}}$ & $6,3 \pm 0,4^{\mathrm{bB}}$ & $7,5 \pm 0,0^{\mathrm{aB}}$ & $0,1 \pm 0,0^{\mathrm{aA}}$ & $28,0 \pm 0,0^{\mathrm{aA}}$ & $28,0 \pm 0,0^{\mathrm{aA}}$ \\
\hline
\end{tabular}

(1) Valores seguidos pela mesma letra não diferem entre si pelo teste de F, a $5 \%$ de probabilidade. ${ }^{\mathrm{a}-\mathrm{b}} \mathrm{C}$ Comparação entre diferentes horários dentro da mesma semana. ${ }^{\mathrm{A}-\mathrm{B}}$ Comparação entre mesmo horário nas diferentes semanas. 
Tabela 3 - Valores médios \pm desvio das variáveis de qualidade de água durante 0 cultivo de tambaqui em canais de abastecimento, em diferentes períodos do experimento ${ }^{(1)}$.

\begin{tabular}{lll}
\hline $\begin{array}{l}\text { Período de } \\
\text { monitoramento (dias) }\end{array}$ & $\begin{array}{l}\text { Condutividade elétrica } \\
\left(\mu \mathrm{S} \mathrm{cm}^{-1}\right)\end{array}$ & $\begin{array}{l}\text { Sólidos dissolvidos totais } \\
\left(\mathrm{mg} \mathrm{L}^{-1}\right)\end{array}$ \\
\hline 5 & $0,1 \pm 0,0^{\mathrm{a}}$ & $18,4 \pm 0,6^{\mathrm{a}}$ \\
25 & $106,0 \pm 0,0^{\mathrm{b}}$ & $53,0 \pm 0,0^{\mathrm{b}}$ \\
40 & $95,7 \pm 0,6^{\mathrm{b}}$ & $48,0 \pm 0,0^{\mathrm{b}}$ \\
\hline
\end{tabular}

(1) Valores seguidos pela mesma letra não diferem entre si pelo teste de Tukey, a $5 \%$ de probabilidade. ${ }^{\mathrm{a} b} \mathrm{C}$ comparação entre a variável nos diferentes períodos.

Tabela 4 - Valores médio \pm desvio dos parâmetros de desempenho de tambaqui cultivados em canais de abastecimento em diferentes densidades após 45 dias de experimento ${ }^{(1)}$.

\begin{tabular}{lccc}
\hline \multirow{2}{*}{ Variáveis observadas } & \multicolumn{3}{c}{ Densidades (peixes m ${ }^{-3}$ ) } \\
\cline { 2 - 4 } & 60 & 90 & 120 \\
\hline Peso inicial (g) & $13,60 \pm 4,10$ & $14,40 \pm 4,90$ & $15,30 \pm 5,30$ \\
Peso final (g) & $50,21 \pm 5,24$ & $46,79 \pm 2,14$ & $50,65 \pm 3,25$ \\
$\begin{array}{l}\text { Ganho de peso médio } \\
\text { por peixe (g) }\end{array}$ & $35,76 \pm 4,64$ & $32,95 \pm 2,38$ & $36,25 \pm 3,25$ \\
$\begin{array}{l}\text { Conversão alimentar } \\
\text { aparente }\end{array}$ & $0,96 \pm 0,10$ & $1,05 \pm 0,06$ & $0,95 \pm 0,08$ \\
$\begin{array}{l}\text { Crescimento específico } \\
\text { em peso (\%) }\end{array}$ & $3,11 \pm 0,24$ & $2,95 \pm 0,11$ & $3,13 \pm 0,15$ \\
Sobrevivência (\%) & $71,80 \pm 4,23$ & $79,12 \pm 3,35$ & $72,5 \pm 4,30$ \\
\hline
\end{tabular}

(1) Não foram detectadas diferenças significativas nos parâmetros zootécnicos em relação aos tratamentos, pelo teste de Tukey, a 5\% de probabilidade.

Os resultados mostraram que é possível que valores mais altos de temperaturas alcançados no período da tarde possam implicar em aumento da ingestão de raçấo neste horário, para suprir a exigência alimentar em decorrência da aceleraçáo do metabolismo destes organismos, haja vista que variaçôes na temperatura modificam as respostas biológicas em peixes, principalmente sobre o ritmo diário de alimentaçáo (Soares e Araújo-Lima 2003; Araújo-Lima e Gomes 2010; Mendonça et al. 2009). Os menores valores desta variável possivelmente ocorreram devido à intensificação do regime de chuvas na calha do Rio Sáo Francisco e na regiáo de captação da água do canal. Contudo, a amplitude de variação da temperatura nas secçốes de cultivo náo foi significativa (máximo de 1,6 ${ }^{\circ} \mathrm{C}$ ) mantendo-se dentro da faixa ótima de confinamento para a espécie (Mendonça et al. 2009).

Em situação de cultivo semelhante a este estudo, Lima et al. (2008) encontraram valores de oxigênio próximos (5,0-6,0 $\left.\mathrm{mg} \mathrm{L}^{-1}\right)$, enquanto que valores inferiores $\left(1,72-3,48 \mathrm{mg} \mathrm{L}^{-1}\right)$ foram encontrados por Oliveira et al. (2007) em sistemas de cultivo com baixo fluxo de água. Estas afirmaçôes sugerem que sistemas de canais (fluxo contínuo de água) melhoram substancialmente as condiçóes de oxigênio dissolvido devido proporcionar melhor renovação da coluna de água.

Apesar do ambiente de confinamento mostrar-se bastante oxigenado, este declinou nas duas maiores densidades de cultivo (90 e 120 peixes $\mathrm{m}^{-3}$ ), atingindo valores de $6,1 \mathrm{mg}$ $\mathrm{L}^{-1}$, provavelmente decorrente do aumento da biomassa e da quantidade de raçáo fornecida. Resultados semelhantes foram encontrados por Maeda et al. (2006) e Lima et al. (2008) monitorando variáveis hídricas em sistemas com alta renovação de água (raceways ) e canais de irrigaçáo com cultivo de tilápias, onde teores de oxigênio chegaram a atingir valores de $3,5 \mathrm{mg} \mathrm{L}^{-1}$ na densidade de 30 peixes $\mathrm{m}^{-3}$, levando a sugerir que podem ser influenciados pela maior densidade e pelo tempo de residência da água.

Os dados de $\mathrm{pH}$ podem ser explicados pelo aumento dos níveis de $\mathrm{CO}_{2}$ e diminuição do ritmo fotossintético durante o período noturno, favorecendo quedas da produção de oxigênio, devido o aumento da respiração por seres autotróficos (Sipaúba-Tavares 1994). Assim, mesmo havendo renovação constante da água em canais, esta não é suficiente para evitar a formação de compostos ácidos. A elevação do $\mathrm{pH}$ também pode estar relacionada à evaporaçáo que ocorre em ambientes como os canais (Redding e Midlen 1992).

Valores de $\mathrm{pH}$ menores encontrados em maiores densidades também ocorreram nos estudos de Lima et al. (2008), em um sistema com fluxo contínuo de água, enquanto que Oliveira et al. (2007) e Brandão et al. (2004) observaram esta mesma condição em sistemas de baixa vazão. Devido o aumento da densidade, há um incremento na taxa de respiração e nos níveis de $\mathrm{CO}_{2}$ no ambiente, o qual, quando em contato com a água, forma o ácido carbônico, favorecendo a diminuição do $\mathrm{pH}$ (Sipaúba-Tavares 1994). Por outro lado, os valores de $\mathrm{pH}$ entre 6,8 e 7,5 não criou condiçôes químicas adequadas para formação da amônia não ionizada, que é tóxica para muitas espécies e manteve-se dentro dos limites aceitáveis para criatórios de peixes (Aride et al. 2007).

A amônia manteve-se dentro dos níveis toleráveis para criaçâo de tambaqui (Chagas et al. 2007). Resultados diferentes foram encontrados por Oliveira et al. (2007), com aumento dos teores de amônia em maiores densidades de cultivo. Estes resultados sugerem que os níveis de amônia em sistema de cultivo em canais são influenciados pelo tempo de cultivo.

O declínio da alcalinidade pode estar associado à diluição das bases pela captação de água com diferentes propriedades limnológicas, aumento do regime de chuvas na calha principal do rio e ao processo de decomposição da matéria orgânica. Apesar deste pequeno declínio observado no período final do estudo, os níveis de alcalinidade foram mantidos dentro dos limites aceitáveis, com um adequado poder tampão, para um bom desempenho do peixe (20 a $300 \mathrm{mg} \mathrm{L}^{-1}$ ) (SipaúbaTavares 1994).

Os dados de dureza contrastam com os encontrados por Lima et al. (2008), que concluiu que o declínio decorrente da dureza foi ocasionado pelo aumento da vazáo do canal ou 
pela presença de moluscos gastrópodes, condição esta não observada neste presente experimento.

Os resultados mostraram que a dureza manteve-se dentro dos limites favoráveis ao cultivo de peixes de água doce (0$75 \mathrm{mg} \mathrm{L}^{-1}$ ), sendo classificada como água com baixos níveis de dureza (Sipaúba-Tavares 1994). Em ambientes aquáticos naturais, a dureza geralmente se equipara à alcalinidade, porque tanto o cálcio $\left(\mathrm{Ca}^{+}\right)$como o magnésio $\left(\mathrm{Mg}^{+}\right)$ encontram-se associados aos íons bicarbonatos e carbonatos, no entanto em águas onde a dureza supera a alcalinidade, parte dos íons $\mathrm{Ca}^{+}$e $\mathrm{Mg}^{+}$se encontram associados a sulfatos, nitratos, cloretos e silicatos, o que não é desejável para os peixes (Sipaúba-Tavares 1994). Dessa maneira, o efeito da evaporação em canais pode ter favorecido o aumento nos teores de dureza, pela possível elevação da quantidade de sais na água, que tendem a precipitar os carbonatos (Redding e Midlen 1992).

Os resultados de condutividade elétrica encontram-se dentro dos limites previstos em cultivos $\left(60 \mathrm{a} 500 \mu \mathrm{cm}^{-1}\right)$, contudo a elevação dos seus níveis ao longo do estudo pode estar associada ao aumento da matéria orgânica na água, proveniente das excretas dos peixes e resto de raçáo não consumida, contribuindo para o acúmulo de íons no ambiente de cultivo (Ituassu et al. 2004); tempo de residência da água ou aumento dos teores de dureza (Oliveira et al. 2010).

Os sólidos dissolvidos totais indicam boa qualidade da água para a piscicultura, uma vez que valores acima de $200 \mathrm{mg}$ $\mathrm{L}^{-1}$ podem ser prejudiciais ao desenvolvimento zootécnico dos peixes. Foram considerados dentro da faixa de conforto para o cultivo de peixes de água doce (5-200 $\mathrm{mg} \mathrm{L}^{-1}$ ) (Wedemeyer 1997).

Na literatura, é comum notarmos densidades de cultivo variadas de acordo com a biologia, idade e fase de cultivo dos peixes (Maeda et al. 2006; Oliveira et al. 2007; Pedreira et al. 2010). No entanto, protocolos para cultivo de peixes, em especial o tambaqui em sistemas de canais, quando se trata de densidade ideal em qualquer fase de cultivo, ainda são incipientes.

Dados obtidos por Arbeláez-Rojas et al. (2002) em sistemas alta vazão (canal de igarapé) indicaram similaridades com o presente estudo. Segundo estes autores os valores médios de ganho de peso obtiveram ganhos de $1,05 \mathrm{~g} \mathrm{dia}^{-1}$ para a mesma espécie, alimentados com raçáo comercial $(30 \%$ de proteína bruta). Estes mesmos autores observaram valores superiores $\left(4,5 \mathrm{~g} \mathrm{dia}^{-1}\right) \mathrm{em}$ viveiros. Este fato pode reforçar a hipótese que, em sistemas com alto fluxo de água, a espécie obteve maior gasto energético devido movimentos natatórios contra o fluxo de água, o que pode ocasionar diminuição no ganho de peso.

A conversão alimentar aparente obtida neste estudo foi melhor do que a reportada por Arbeláez-Rojas et al. (2002)
(CAA 1,80), Brandáo et al. (2004) (CAA 1,27-0,92) com juvenis desta espécie, Brandão et al. (2005) (CAA 1,35-1,31) avaliando a densidade de juvenis de matrinxá durante a recria em tanques redes e finalmente, por Maeda et al. (2006) (CAA 1,56-1,30), na segunda alevinagem de tilápia nilótica em sistemas de cultivo com alta renovação de água. Os resultados demonstram que juvenis de tambaquis são táo eficientes em converter a ração em peso quanto à tilápia nilótica e matrinxã em diferentes sistemas de cultivo. É possível que o ritmo diário de alimentaçáo de três vezes ao dia empregado no cultivo, tenha favorecido os juvenis, traduzindo em um melhor manejo alimentar (Fernandes et al. 2010).

No presente estudo, as variáveis de desempenho; ganho de peso e conversáo alimentar aparente em diferentes tratamentos se mantiveram no mesmo padráo com o aumento da quantidade de indivíduos, e, portanto, dentro do patamar ideal para criação de espécies onívoras (Kubitza 2004).

A piscicultura moderna se baseia em sistemas de produção onde ocorram múltiplas funçôes do uso da água (Subasinghe $e t$ al. 2009). Esta afirmação abre precedentes para que canais de abastecimento se tornem ótimos sistemas para a manutençáo do ambiente aquático, uma vez que se aproveita uma estrutura já existente, com fluxo contínuo de água, por onde pode haver um incremento nas densidades de cultivo e com múltiplas funções (piscicultura e irrigação de culturas), obtendo máxima produtividade por área e um pescado com níveis de proteína mais desejável para indústria de processamento e beneficiamento.

A sobrevivência foi inferior a obtido por Brandão et al. (2004) (80\%), durante a recria de juvenis de tambaqui em tanques-rede, e por Arbeláez-Rojas et al. (2002) (96\%), no cultivo de tambaqui em canais de igarapé, e superior a encontrada por Maeda et al. (2006) (60\%), no cultivo de tilápia nilótica em sistema de alta renovação (raceway). A mortalidade pode ser atribuída às condiçôes do ambiente aos quais juvenis de tambaqui foram submetidos, uma vez que, esta espécie, quando jovem, prefere ambientes lênticos à lóticos (Araújo-Lima e Goulding 1998; Araújo-Lima e Gomes 2010). A presença do bacilo gran negativo Flavobacterium columnare encontrados nas brânquias de boa parte dos exemplares debilitados, sugerem que estes podem ter contribuído com o aumento da taxa de mortalidade, entretanto esta hipótese não foi testada no presente experimento.

\section{CONCLUSÕES}

$\mathrm{O}$ uso de canais de irrigaçáo com a finalidade de cultivo de tambaquis é uma alternativa de custo barato nas regióes do semiárido nordestino por manter boa qualidade ambiental e padróes de água adequados para o multiuso. 


\section{AGRADECIMENTOS}

Ao fundo de Amparo a pesquisa do Estado de Alagoas (FAPEAL) e a Companhia de Desenvolvimento dos Vales do São Francisco e do Parnaíba (CODEVASF).

\section{BIBLIOGRAFIA CITADA}

American Public Health Association (APHA). 1995. Standard methods for the examination of water and wastewater. 19 ed. Washington, DC, 1995, 1134p.

Araújo-Lima, C. A. R. M.; Gomes, L. de C. 2010. Tambaqui (Colossoma macropomum). In: Baldisserotto, B.; Gomes, L. de C. Espécies nativas para piscicultura no Brasil. 2da Ed. Santa Maria: UFSM, p.175-204.

Araújo-Lima, C. A. R. M.; Goulding, M. 1998. Os frutos do Tambaqui: ecologia, conservação e cultivo na Amazônia. Sociedade Civil Mamirauá, Brasília, 1998, 186p.

Arbeláez-Rojas, G. A.; Fracalossi, D. M.; Fim, J. D. I. 2002. Composição corporal do tambaqui, Colossoma macropomum e matrinxá, Brycon cephalus, em sistema de cultivo intensivo, igarapé e semi-intensivo, viveiros. Revista Brasileira de Zootecnia, 31: 1059-1069.

Aride, P. H. R.; Roubach, R.; Val, A. L. 2007. Tolerance response of tambaqui Colossoma macropomum (Cuvier) to water $\mathrm{pH}$. Aquaculture Research, 38: 588-594.

Brandão, F.R.; Gomes, L.C.; Chagas, E.C.; Araújo, L.D. 2004. Densidade de estocagem de juvenis de tambaqui durante a recria em tanques-rede. Pesquisa Agropecuária Brasileira, 39: 357-362.

Brandão, F.R.; Gomes, L.C.; Chagas, E.C.; Araújo, L.D.; Silva, A.L.F. 2005. Densidade de estocagem de juvenis de matrinxã (Brycon amazonicus) durante a recria em tanques-rede. Pesquisa Agropecuária Brasileira, 40: 299-303.

Chagas, E.C.; Gomes, L.C.; Martins Júnior, H.; Roubach, R. 2007. Produtividade de tambaqui criado em tanque-rede com diferentes taxas de alimentaçáo. Ciência Rural, 37: 1109-1115.

Fernandes, T. R. C.; Doria, C. R. C.; Menezes, J. T. B. 2010. Características de carcaça e parâmetros de desempenho do tambaqui (Colossoma macropomum, Cuvier 1818) em diferentes tempos de cultivo e alimentado com raçôes comerciais. Boletim do Instituto de Pesca, 36: 45-52.

Ituassu, D.R.; Santos, G.R.S.; Roubach, R.; Pereira-Filho, M. 2004. Desenvolvimento de tambaqui submetido a períodos de privação alimentar. Pesquisa Agropecuária Brasileira, 39: 1199-1203.

Kubitza, F. 2004. Coletânea de informaçóes aplicadas ao cultivo do tambaqui, do pacu e de outros peixes redondos. Panorama da Aqüicultura, 14: 27-37.

Lima, C.B.; oliveira, E.G.; Araújo-Filho, J.M.; Santos, F.J.S.; Pereira, W.E. 2008. Qualidade da água em canais de irrigação com cultivo intensivo de tilápia nilótica (Oreochromis niloticus). Revista Ciências Agronômicas, 39: 531-539.

Maeda, H. Silva, P. C.; Aguiar, M. S.; Pádua, D. M. C.; Oliveira, R. P. C. 2006. Efeitos da densidade de estocagem na segunda alevinagem de tilápia nilótica (Oreochromis niloticus) em sistema raceway. Ciência Animal Brasileira, 7: 265-272.
Mendonça, P.P.; Ferreira, R.A.; Vidal Junior, M.V.; Andrade, D.R.; Santos, M.V.B.; Ferreira, A.V.; Rezende, F.P. 2009. Influência do fotoperíodo no desenvolvimento de juvenis de Tambaqui (Colossoma macropomum). Archivos de Zootecnia, 58: 323-331.

MPA - Ministério da Pesca e Aquicultura, 2010. Produção pesqueira e aquícola: Estatistica 2008 e 2009. 29p. (http://www.mpa.gov. $\mathrm{br} / \mathrm{mpa} /$ seap/Jonathan/mpa3/dados/2010/Docs/Caderno\%20

Consolida \%C3\%A7\%C3\%A3o\%20dos\%20dados \% 20 estatiscos\%20final\%20curvas\%20-\%20completo.pdf). Acesso em 18/01/2011.

Oliveira, R.P.C.; Silva, P.C.; Paduas, D.M.C.; Aguiar, A.; Maeda, H.; Machado, N.P.; Rodrigues, V.; Silvas, R.H. 2007. Efeitos da densidade de estocagem sobre a qualidade da água na criação do tambaqui (Colossoma macropomum, Cuvier, 1818) durante a segunda alevinagem, em tanques fertilizados. Ciência Animal Brasileira, 8: 705-711.

Oliveira, R.P.C.; Silva, P.C.; Brito, P.P.; Gomes, J.P.; Silva, R.F.; Silveira Filho, P.R.; Roque, R.S. 2010. Variáveis hidrológicas físico-químicas na criação da tilápia-do-nilo no sistema raceway com diferentes renovações de água. Ciência Animal Brasileira, 11: 482-487.

Pedreira, M.M.; Sampaio, E.V.; Santos, J.C.E.; Luz, R.K. 2010. Cultivo de matrinxã Brycon orthotaenia (Günther, 1864) em tanques-rede, em diferentes densidades de estocagem. Acta Scientiarum. Biological Sciences, 32: 17-22.

Pereira Filho, M., Fonseca, F.A.L., Silva, J.A.M. E Brandão, L.V. 2008. Nutrição e boas práticas de manejo em aqüicultura. PUBVET, (on line), 2(18). (http://www.pubvet.com.br/ artigos_det.asp?artigo=384). Acesso em 18/01/2011.

Redding, T. A.; Midlen, A. B. 1992. Estudio de la producción piscícola en los canales de riego. FAO Documento Técnico de Pesca. no 317, Roma, 1992, 114p. (http://www.fao. org/DOCREP/003/T0401s/T0401s00.HTM). Acesso em $18 / 01 / 2011$

Sipaúba-Tavares, L. H. 1994. Limnologia Aplicada a Aqüicultura. Jaboticabal: FUNEP, 1994, 70p.

Soares, E. C. S.; Lima, C. A. R. M. A. 2003. Influencia do Tipo do alimento e da temperatura na Evacuação Gástrica da Piranha caju (Pygocentrus nattereri). Acta Amazonica, 34: 35-45.

Subasinghe, R.; Soto, D.; Jia, J. 2009. Global aquaculture and its role in sustainable development. Reviews in Aquaculture, 1: $2-9$.

Vallejo, S.V; González-Posada, J. O. 2007. Acuicultura: La revolución azul. Publicaciones científicas y tecnológicas del Observatorio Español de Acuicultura. Madrid, 2007, 363p.

Wedemeyer, G.A. 1997. Effects of rearing conditions on the health and physiological quality of fish in intensive culture. In: Iwana, G.K,; Pickering, A.D.; Sumpter, J.P; Schreck, C.B (Ed.). Fish stress and health in aquaculture. Cambridge University Press, United Kingdom, p. 35-71.

Recebido em: 08/10/2012

Aceito em: 29/01/2013 
